\title{
Tasas óptimas para el Impuesto a la Minería del Cobre en Chile
}

\author{
Jan Cademartori D. \\ Universidad Católica del Norte, Antofagasta, Chile. Email: jcademar@ucn.cl \\ Carlos Paéz S. \\ Universidad Católica del Norte, Antofagasta, Chile. Email: cpaezucn@gmail.com

\section{Juan Daniel Soto D.} \\ Universidad Católica del Norte, Antofagasta, Chile. Email: juan.soto@msn.com
}

\begin{abstract}
Resumen: En Chile existen fuertes demandas sociales. Sin embargo, existe falta de investigación académica con respecto a la cuantía de posibles fuentes de mayores ingresos fiscales. En este trabajo se propone un nivel de impuesto a las compañías mineras multinacionales que permitiría traspasar al Estado sus ganancias sobre-normales. Además, se estima el valor de estos beneficios. Ambas estimaciones utilizan el método de valor presente neto para representar la rentabilidad de un proyecto de minería de cobre de tamaño medio. Los resultados se analizaron utilizando diferentes precios del cobre y de otros parámetros del modelo.

Palabras clave: tributación minera, empresas multinacionales, renta económica, teoría de la dependencia.
\end{abstract}

\section{Optimal taxes rates for copper mining in Chile}

Abstract: In Chile there are strong societal demands. However, there exists a lack of scholarly research regarding possible sources of fiscal revenue for social investment. This paper proposes taxes on multinational mining companies that would allow to transfer to the state their above-normal returns. Additionally, this work estimates the value of these profits. Both estimates use the net present value method to represent the profitability of an average copper mining project. The results were tested using varying copper prices and other model parameters.

Key words: mining taxation, multinational companies, economic rent, dependency theory.

\section{Taxas de imposto ideais para mineração de cobre no Chile}

Resumo: No Chile existem fortes demandas sociais. No entanto, falta de pesquisa acadêmica sobre a quantidade de potenciais fontes para um aumento das receitas fiscais. Neste trabalho é proposto um nível de imposto para as empresas de mineração multinacionais que permitiria transferir para o Estado seus ganhos além de um patamar normal. Além disso, é estimado o valor desses benefícios. Ambas as estimativas usam o método do valor presente líquido para representar a rentabilidade 
de um projeto de mineração de cobre de tamanho médio. Os resultados foram analisados utilizando diferentes preços do cobre e de outros parâmetros do modelo.

Palavras-chave: tributação da mineração, empresas multinacionais, renda econômica, teoria da dependência.

$* * *$

\section{Introducción}

La tributación minera en Chile ha generado gran debate desde que el sector privado tiene un volumen absoluto y una participación creciente en la producción de cobre. En un sector además que representa por si solo más del 50\% de las exportaciones de país. Dada su importancia, los recursos del cobre pueden servir para atacar las grandes desigualdades sociales internas y para corregir la especialización de la economía en materias primas. Para obtener un mayor ingreso fiscal del cobre, existe un amplio abanico de alternativas; aquí se analiza la tributación privada.

Hay que recordar que después del golpe de estado de 1973, la dictadura militar, si bien mantuvo las minas nacionalizadas en manos de la empresa estatal CODELCO, creó una legislación favorable a las empresas extranjeras. Estas volvieron masivamente a Chile después de acabado el régimen militar en 1990. Desde entonces, CODELCO amplió ligeramente su producción, pero los capitales privados la superaron ampliamente, al punto que actualmente estos representan cerca de dos tercios de la producción obtenida de territorio chileno (Gráfico 1).

\section{Gráfico 1: Producción de cobre desde Chile 1990-2011. Producción privada versus estatal. En miles de toneladas métricas.}

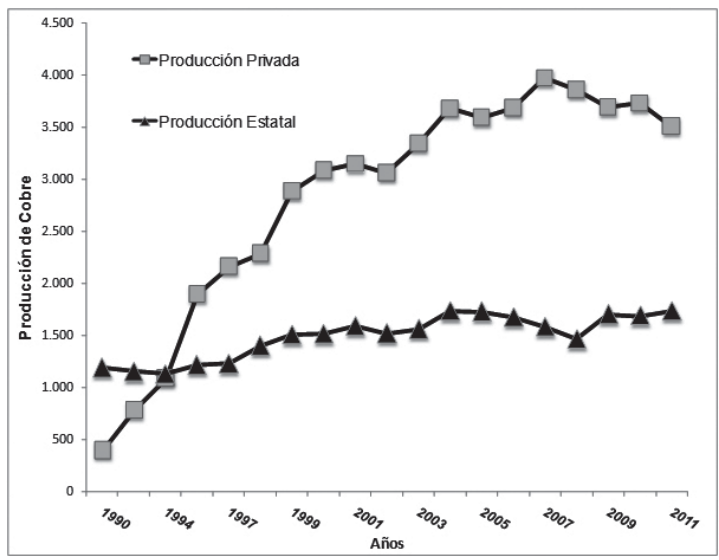

Fuente: Elaboración propia en base a COCHILCO. 
Esta producción privada tiene varias características. Primero, los inversionistas son principalmente extranjeros (Cuadro 1 en anexo) y hasta el grupo nacional que participa de modo importante (Antofagasta Minerals) tiene su casa matriz en el exterior. De este modo las ganancias se exportan masivamente. Segundo, la producción privada se compone en gran parte de productos con bajo valor agregado, particularmente concentrado de cobre que contiene menos de $40 \%$ de contenido de este metal. Además los bienes de capital y la energía que necesita la minería provienen principalmente de empresas de propiedad extranjera. Asimismo, la minería del cobre se encuentra concentrada en las regiones del Norte de Chile (Arica, Tarapacá, Antofagasta, Atacama), en el desierto de Atacama, donde el agua, que es un factor productivo clave en la gran minería, casi se encuentra agotada. Finalmente, las grandes empresas han venido utilizando una mayoría de trabajadores subcontratados que no tienen mayores beneficios sociales (Cuadro 1 anexo y Leiva (2009). De lo anterior se concluye que la minería extranjera conforma aún una suerte de economía de enclave.

En este contexto, no resulta extraño que se discuta la forma de capturar una mayor parte de la renta minera. Desde ya, Chile presenta una carga tributaria inferior a lo que cabría esperar de acuerdo a su nivel de ingreso (CEPAL, 2012: Gráfico IV.2) y con baja participación de los impuestos a la renta que son los que permiten cobrar más a las personas y empresas de mayor ingreso (Ferreira, Messina, Rigolini et al., 2013: Gráfico 6.7). Esta situación se extiende a la minería donde, como se muestra más adelante, se obtienen ganancias sobre-normales con los impuestos que actualmente existen. Más aún si hubiere proyectos mineros por 100 mil millones de dólares en los próximos diez años, según las autoridades del sector.

Este trabajo comienza analizando la legislación tributaria chilena que afecta a la minería multinacional. Esto permite contextualizar el modelo de análisis que se presenta en la sección siguiente. Se pretende con éste modelo analizar si hay holguras para incrementar la carga tributaria a la minería multinacional. Se obtiene además la rentabilidad que están obteniendo actualmente las empresas privadas y la magnitud de la renta desaprovechada por el Estado. Finalmente, los resultados se evalúan haciendo cambiar el valor de algunos parámetros claves del modelo tales como el precio del cobre y los costos de las empresas.

\section{Legislación tributaria en la minería privada chilena}

Los tributos afectos en la minería privada multinacional, son impuestos a las ganancias declaradas, el resto, son marginales. Las empresas exportadoras reciben de regreso el IVA por sus compras, tributo que es la principal fuente de recaudación fiscal del país. Salvo para algunos pequeños municipios mineros, tampoco es significativa la patente de explotación por hectárea minera, ni el derecho de aduana a los bienes importados, que en Chile alcanza un nivel promedio cercano a cero en virtud de la política comercial. Incluso el impuesto por consumo de combustibles es devuelto 
en la medida que se use dentro de las faenas. De igual forma, el consumo de agua tampoco paga tributo, incluso aunque el agua sea comercializada hacia terceros. Hay que agregar además que las empresas en Chile no cotizan a la seguridad social ya que los fondos de pensiones son financiados íntegramente por los mismos trabajadores.

Hay tres impuestos a las ganancias: el impuesto general a las utilidades, el impuesto sobre las remesas de utilidad remitidas al exterior del país y el impuesto específico a la minería. El impuesto general a las ganancias (Primera Categoría) afecta a todos los sectores productivos y grava con una tasa del $20 \%$ a las utilidades repartidas, la cual hasta hace poco fuera del 17\%. A continuación aparece el impuesto adicional. Afecta solo a las remesas de ganancias exportadas fuera del país y formalmente alcanza a un 35\% de éstas. Como el impuesto general se considera avance de impuesto adicional, en la práctica, la tasa efectiva del impuesto adicional es de tan solo del orden de $16 \%$.

Hay que advertir que este impuesto adicional, más que un tributo a la empresa, reemplaza al gravamen (Global Complementario) que el dueño de la empresa habría asumido como persona natural en Chile. Este punto es importante si se desea comparar empresas y no personas; los impuestos personales aplican sobre la suma de ingresos obtenidos por diferentes conceptos, en diferentes sectores y lugares del planeta. Esto hace muy complejo comparar la carga chilena con la internacional. Más aún, teniendo en cuenta que los impuestos a los ingresos personales están sujeto a una tasa porcentual que aumenta con los ingresos declarados por la persona, es decir, a una tasa progresiva.

El tercer gravamen es el Impuesto Específico a la Minería (IEM). Después de varios intentos frustrados, y bajo presión ciudadana, la primera versión de IEM se aprobó en el año 2005 con la Ley N²0.026. Este tributo no es propiamente un royalty, en el sentido que su tasa (\%) se aplique sobre el valor de la producción extraída ni sobre las ventas. El IEM es una tasa sobre la Renta Imponible Operacional Minera(RIOM), que son los ingresos de la actividad minera menos sus costos de producción (materias primas, energía, mano de obra y otros), la depreciación financiera del equipo de capital y los intereses financieros, que se van añadir en una ley posterior. Al considerarse los costos, se genera el problema de la credibilidad de los costos informados por cada empresa, tema que será analizado más adelante.

En esta primera versión de IEM, se estableció una tasa variable según la producción, con máximo del 5\% que es la más común para la minería multinacional. En la segunda versión, actualmente vigente, rige la Ley N²0.469 del año 2010, aprobada con ocasión del terremoto que afectó al país. Si bien se mantuvo la idea de aplicar una tasa sobre la RIOM, cambió el valor de las tasas. Asimismo cambió su dependencia de la producción, al establecerse tasas que son función del margen operacional minero (MOM) que representa la participación del RIOM en las ventas de cada empresa. 
Por ejemplo, una empresa que tuvo un MOM de $50 \%$, significa que generó un ingreso neto después de pagar sus costos corrientes, de 50\% de sus ventas y por tanto le corresponde pagar un impuesto específico IEM de aproximadamente 5,9\% de su RIOM medida en pesos (Cuadro 2 en Anexo). A mayor MOM, mayor tasa sobre la RIOM. Estas aumentaron entre los años 2010 y 2012, y vuelven a incrementarse posteriormente, alcanzando un tope cercano a $14 \%$ que en realidad se transforma en $12,3 \%$ antes del mismo impuesto IEM.

Sin embargo, ninguna de estas dos leyes ha tenido gran incidencia tributaria. Primero, porque comparando los tres impuestos citados, el IEM es el de menor recaudación fiscal dadas sus menores tasas (12,3\% máximo) en comparación a los dos restantes (20\% y 16\% parejos). Segundo, porque perduran cláusulas de estabilidad tributaria que permiten mantener la tributación previa por muchos años. Tercero, quizás lo más importante, por que persisten mecanismos de evasión legal (elusión tributaria) y probablemente de evasión ilegal que afectan a los tres tipos de impuestos.

Respecto a lo segundo, se debe aclarar que el Estado chileno firma con la inversión extranjera un convenio llamado de invariabilidad tributaria que no rige para el resto de las empresas ni personas chilenas. Este contrato garantiza la carga a cambio de un pequeño porcentaje extra en la tasa de impuestos. Una vez firmado, para convencer al inversionista a renunciar a este acuerdo, los gobiernos han ofrecido alzas pequeñas contra mayor estabilidad. Los inversionistas generalmente lo aceptan para prevenir un cambio más de fondo en el marco institucional. Estas garantías son discutidas por quienes consideran que se viola de este modo el principio constitucional de igualdad ante la ley y aquel otro donde el Estado solamente concesiona las minas que son de su propiedad.

Así por ejemplo, la reforma citada del año 2005, otorgó un nuevo período de estabilidad por 12 o por 15 años según el caso, a cambio de someterse al IEM de 4\% o de 5\%. Sin embargo, el Estado rebajó la tasa adicional, que como se explicó antes, es más gravosa que el IEM. Entonces las empresas aceptaron el IEM porque aumentó la carga apenas en 1\% de sus ventas según nuestra estimación, a cambio de lo cual prologaron la estabilidad tributaria por más de una década. Además, se brindaron descuentos adicionales por lo primeros años de aplicación.

La citada Ley 20.469, del año 2010, complicó aún más el panorama de estabilidad tributaria. Algunas empresas pueden mantenerse con el antiguo IEM amparadas en la estabilidad anterior. Para otras, la estabilidad se extiende por otros nueve años de ser aceptada las nuevas tasas. Sin embargo, las empresas podían aceptar parcialmente esta reforma, sometiéndose únicamente a las tasas de los años 2010-2012 de la reconstrucción, para retomar el IEM del 5\% por el sobrante de seis años de estabilidad en que las dejó la ley del año 2005. Respecto a las empresas que se acogen a las tasas de después de 2012, no queda claro si estas rigen inmediatamente a partir de 2013, o después de expirar la invariabilidad tributaria anterior. La 
enorme gama de posibilidades al parecer se resuelve por medio de dictámenes interpretativos del Servicio de Impuestos Internos.

Respecto a lo segundo, estas reformas de los años 2005 y 2010, no atacaron el problema de elusión tributaria. Para calcular la carga efectiva, deben considerase los múltiplos medios con que cuenta la minería privada, sobre los cuales hay denuncias desde la sociedad civil (Alcayaga, 2005) y desde el Parlamento. Por ejemplo Meller (2002) recuerda que el Servicio de Impuestos Internos, al ser consultado por una Comisión Investigadora del Senado, entregó cifras de recaudación muy inferiores a las que publican las empresas privadas en sus informes financieros.

En ello, resulta central la declaración de ingresos por sub-productos mineros que se exportan junto al cobre, tales como el molibdeno, el oro, la plata y otros. Riesco y García Huidobro (2010) resaltan que es difícil explicar que CODELCO, con minas de casi un siglo de existencia, presente en sus informes financieros una mayor rentabilidad por tonelada (antes de impuestos) que la minera privada más grande del país, con menos de diez años de antigüedad, y con modernas inversiones. La explicación principal estaría en la sub-declaración de ingresos por sub-productos. A este respecto, se ignora la efectividad del Artículo 5 de la misma Ley N²0.469 .que obliga al Servicio Nacional de Aduanas a examinar la composición de las exportaciones de concentrados de cobre y sus subproductos (Ag, Au, Mb, entre otros); dicho análisis debería cubrir entre un 3\% y 10\% del total de las exportaciones de concentrado. Se sabe en cambio que esta labor es concesionada a laboratorios privados.

En cuanto a los medios legales, las empresas cuentan para rebajar impuestos, entre otros, con los intereses de su deuda. En Chile, las empresas extranjeras de la minería, comienzan con altos porcentajes de endeudamiento. Ello no solo les permite rebajar el gravamen general (ya que los intereses son un gasto que disminuye la renta sujeta al impuesto de primera categoría). Al remitir sus utilidades al exterior, bajo la forma de pago de intereses, el inversionista extranjero puede gozar de un tributo de apenas $4 \%$ en lugar del $16 \%$ de la mencionada tasa adicional. Este asunto se hizo más visible al momento de la venta en el año 2002, de una mina de Exxon (La Disputada Las Condes) en 1.300 millones de dólares, la cual estuvo sin pagar impuestos de primera categoría durante 23 años porque no registraba ganancias tributables (González, 2004). La ley 19.738 del año 2002, llamada contra la elusión tributaria, no cambió mucho esta situación al colocar el tope de endeudamiento de las empresas extranjeras en un 75\% de sus bienes (activos).

La depreciación del capital físico, también permite rebajar de la base imponible como gasto, lo que fue alguna vez aporte de capital. Como se sabe, la depreciación no es un gasto de dinero. Además, la legislación, con el ánimo de subsidiar la compra de equipo, permite la depreciación acelerada del equipo. Siendo la minería un sector intensivo en bienes de capital, la compra del equipo y su subsecuente depreciación pueden ser de gran 
ayuda para reducir y para postergar el pago de impuestos. Más aún si en Chile, los bienes importados están prácticamente exentos de aranceles.

La base tributable igualmente se puede recortar a través de otras herramientas legales, comunes a todos los sectores productivos. La pérdida de un período se arrastra a los períodos siguientes para fines tributarios. Así como la depreciación, del mismo modo se puede acelerar la amortización de los gastos de organización y puesta en marcha. Los resultados negativos en la especulación financiera de futuros igualmente aparecen como gastos necesarios para generar la renta del impuesto general. También reducen impuestos parte de las donaciones y el gasto en capacitación de personal. En la minería multinacional, el arriendo de las minas a otra empresa, se registra como gasto, como también se amortiza la inversión en ampliación de la mina (desarrollo). Las empresas están incluso autorizadas para presentar sus declaraciones tributarias en moneda extranjera.

Con estas ventajas tributarias, no es de extrañar entonces que en las empresas privadas de la gran y mediana minería de Antofagasta, la suma de los impuestos a la renta pagados entre 1987 y 1991, representaban apenas $0,8 \%$ de sus ventas. En el mismo período, estas corporaciones retiraron del país ganancias por $\$ 237.000$ millones mientras declaraban pérdidas en los libros tributarios, por valor de \$3.427.000 millones (Cademartori, 2005)

Por lo anteriormente expuesto, en este trabajo se utiliza una metodología que tiende a subestimar las ganancias efectivas del sector privado, porque los datos de costos provienen de lo informado por las empresas en su Contabilidad Financiera. Además, cada empresa tiene sus propios contratos de invariabilidad tributaria que probablemente le permiten mantener su carga fiscal. La metodología permite en todo caso proponer cual debería ser una tributación mínima con esta limitada información y estimar la pérdida fiscal por no tenerla.

\section{Marco teórico y metodología.}

Para ello se parte del concepto de renta. Se considera renta la ganancia que obtienen las empresas por encima de la necesaria para mantener su actividad. Conseguida ésta de la explotación del cobre, se debe a la renta absoluta derivada de la escasez mundial del recurso y a la renta relativa, generada de la superior calidad geológica de los yacimientos chilenos y de otras características específicas del país, tales como la ubicación de las minas, la infraestructura disponible, el capital humano. Estas características geográficas no se pueden producir y reproducir a través de la actividad humana, como se fabrican automóviles y la mayoría de los bienes y servicios. La renta refleja entonces la escasez mundial de yacimientos de calidad superior, lo cual permite al concesionario, el Estado, de gozar de una suerte de monopolio sobre el recurso natural.

Esto obliga a distinguir entre la renta del propietario del recurso y la 
ganancia del capitalista que recibe la concesión del recurso. En esta metodología se asume que las empresas privadas mantienen el interés por la concesión mientras su ganancia, como proporción del capital invertido, alcance un nivel de acuerdo al riesgo que asumen. El excedente obtenido por encima de esa ganancia no tiene incidencia sobre su nivel de producción reconocida su amplio margen de utilidad operacional, por lo cual puede ser capturado por el Estado.

Una meta razonable sería contar con una carga equivalente a la de otros países mineros, Desgraciadamente, como se indicó antes, el impuesto adicional, sustituye al impuesto a las personas, lo cual crea un problema metodológico difícil de solucionar. Tampoco es evidente la forma de ajustar los convenios para evitar la doble tributación y los subsidios del Estado. Asimismo los costos de producción mineros, varían entre un país y otro, en circunstancia que las empresas privadas explotan en Chile yacimientos de menor costo. Teniendo en cuenta estas precauciones, se hace necesario analizar la carga tributaria efectiva en Chile mediante un modelo de empresa representativa.

La referencia corresponde a un proyecto representativo que dura 21 años de los cuales, los dos primeros son de construcción, sin generación de ingresos y los 19 años siguientes, son de operación a capacidad plena. Se presupone una empresa productora de 250.000 toneladas de cobre fino al año que representa a la mediana minería, parecida al tamaño del proyecto Collahuasi. El modelo usa el promedio de costos de la minería chilena pero el impuesto óptimo que se propone queda en función de la rentabilidad MOM de cada proyecto como lo establece la actual ley.

En el modelo propuesto, se estima como ganancia normal del concesionario, una tasa de rentabilidad del 12\%. Este coeficiente es empleado para obtener el valor presente neto del capital propio en el escenario base. La razón de haber escogido esta tasa de descuento, es que ella fue utilizada en el citado estudio de Otto, Batarseh y Cordes (2000) que compara carga tributaria entre países mineros. Por otro lado, según nuestras fuentes informales, se exige entre $10 \%$ y $12 \%$ en las empresas mineras instaladas en Chile para aceptar un proyecto. Desgraciadamente, la primera es tasa al capital propio mientras que la segunda refleja el costo de capital promedio de la firma. En cualquier caso, se sensibilizan los resultados con diferentes tasas, posibles niveles de precios y de costos.

Las cifras están en dólares de diciembre del año 2009. En el escenario base se ha supuesto un precio promedio anual de la libra de cobre de USS\$2 en moneda constante. Este precio corresponde a la media del último ciclo (2001-2009). Este valor también es el promedio real 1950-2008 deflactado por el IPM de Estados Unidos con base 2008 y medido por COCHILCO (2011) en la Bolsa de Metales de Londres. Por otro lado, como sabemos, el precio de los últimos años ha sido cercano a los US\$ 3. Así, un precio de USS \$ 2 no parece optimista para el escenario base. 
Los datos de costos fueron obtenidos de 52 observaciones de 13 empresas sujetas al Impuesto Específico a la Minería (IEM) entre los años 2006 y 2009. El costo operacional proviene de la regresión econométrica que arroja un costo operacional por unidad variable de $\mathrm{v}=$ 0,6 por libra de cobre y un costo fijo medio de 165 por año que aproxima US $\$=0,3$ por unidad. Por su parte, la depreciación financiera contable (US\$ 0,1 por libra) se estimó también en base a regresión y se asumió como gasto efectivo en reposición de bienes de capital, el cual se financia con las utilidades del proyecto. Estas regresiones presentan coeficientes de ajuste r2 superiores al 80\%. La reposición no incluye la inversión inicial, estimada a base del promedio de inversión por tonelada (US\$ 5,4 mil) de producción final de cobre,de una lista de 13 proyectos de cobre de los últimos años previos a 2010.

Para fines tributarios, se empleó depreciación acelerada de modo que los equipos se amortizan en la mitad de su vida útil. También se aprovecha la ventaja tributaria dela deuda que resulta crucial como se explicó antes. El proyecto se financia en 30\% con capital propio, en 35\% con deuda relacionada y en 35\% con deuda externa. La deuda relacionada se trata como capital propio salvo para establecer la carga tributaria. La deuda cae rápidamente porque se amortiza en cuotas anuales fijas antes del año 11; su tasa de interés es de 8\% anual. Esto refleja el patrón actual.

La carga tributaria óptima se encuentra con el IEM que anula el Valor Presente Neto del proyecto. Se presenta bajo dos alternativas. Como IEM óptimo sobre el RIOM (antes IEM) y como royalty óptimo sobre las ventas. Se puede demostrar que la tasa royalty sobre las ventas, cualquiera sea su nivel r, es igual a la tasa t del IEM antes impuesto, multiplicada por el MOM. En efecto, sean: V: nivel de ventas, R: recaudación fiscal por royalty, T: recaudación fiscal por IEM, MOM: Margen Operacional Minero $(\mathrm{RIOM} / \mathrm{V})$. Imponiendo que: $\mathrm{T}=\mathrm{t}(\mathrm{RIOM})=\mathrm{R}=\mathrm{r}(\mathrm{V})$, se concluye que $\mathrm{r}$ $=\mathrm{t}(\mathrm{MOM})$

La pérdida país por no tener este impuesto, se mide como la diferencia entre los ingresos fiscales que se habrían obtenido con la tasa óptima y los ingresos fiscales actuales, bajo el supuesto que la empresa promedio tiene una carga tributaria IEM del 4\% los primeros 12 años y de $5 \%$ los 7 años restantes, en virtud del arrastre de la estabilidad tributaria. Por otra parte, la pérdida fiscal promedio anual no se ha actualizado a valor presente porque buscar una tasa de descuento llevaría a otro estudio.

La pérdida fiscal se obtuvo multiplicando la pérdida por tonelada del proyecto representativo, por la producción total privada de las empresas privadas sujetas al IEM (3,2 millones TM de fino al año 2009). En otras palabras, a pesar de las diferencias de costos, se asume que ninguna empresa cerraría su planta a causa del impuesto. En primer lugar, porque el impuesto depende de la rentabilidad del precio del cobre. Además, la expe- 
riencia de los últimos veinte años, con precios del cobre debajo de un dólar en ciertos años, pero con ganancias sobre normales la mayor parte del tiempo, hace pensar que los costos de las empresas rara vez superan a sus ingresos.

En la tabla de resultados también se compara el impuesto óptimo IEM* con el impuesto IEM más reciente (IEMx) establecido por la Ley N²0. 469. Para este efecto, fue necesario convertir las tasas después de impuestos que se presenta en los textos legales, en tasas antes de impuesto, que resultan más claras y consistentes con el resto de los impuestos. Nótese que la tasa efectiva antes impuesto que publica el Servicio de Impuestos Internos (Cuadro 2 anexo, columna D) no es el promedio simple de las tasas marginales acumuladas, sino un promedio ponderado donde el primer tramo tiene un significativo peso de $35 \%$ mientras que los siguientes alcanzan un valor máximo de apenas 5\% en el punto final del tramo. Así, el impuesto es menor que asignando a cada tramo igual peso. Además, como se indicó antes, la tasa efectiva antes de impuestos, que se aplica sobre la RIOM (columnas $\mathrm{G}$ o H) es menor a la misma después de impuestos (columna D). De este modo, ambos mecanismos permiten bajar las tasas efectivas.

\section{Resultados}

En el Cuadro 1 se presentan simulaciones para diferentes precios del cobre manteniendo constante el resto de las variables. Se supone que la empresa ha pagado los tres impuestos a la renta como la ley ordena y sin otra elusión que usar deuda relacionada en un 35\% y adelantando la depreciación como lo permite la ley. Una vez escogido el valor de cualquier parámetro, este se mantiene constante durante los 21 años de proyecto. Para determinar el impuesto óptimo, como se explicó antes, se tolera una tasa de rentabilidad privada anual de $12 \%$ después de haber recibido de vuelta el capital pagado y de cubrir todos los egresos del proyecto.

La segunda columna del Cuadro 1 estima la rentabilidad del proyecto (TIR). Por ejemplo, en el escenario base $(\mathrm{P}=2)$, la rentabilidad del $25 \%$, significa intuitivamente que, al final del cuarto año, se pueden financiar dos proyectos del tamaño del proyecto original ya que se ha devuelto íntegramente el capital inicial y en cada año se obtuvo una rentabilidad neta de un cuarto de proyecto. Estas cifras desmienten a Leturia y Merino (2004: Gráfico 4) quienes no encuentran ganancias sobre-normales en la minería trasnacional utilizando informes de las empresas para los años de crisis 2001-2002.

En la tercera columna, se encuentra el Margen Operacional Minera (MOM) que aumenta con el precio del cobre, dado el nivel de costos. Como se indicó antes, según la última Ley, el MOM determina el IEM (IEMx) que corresponde aplicar. En la columna siguiente, $\mathrm{R} *$ exhibe el royalty sobre las 
ventas que capturaría la misma tributación del IEM* como se explicó en la sección anterior. Para que la captura sea idéntica, se debe suponer que la capacidad fiscalizadora del Estado es similar sobre las ventas que sobre los costos, lo cual es improbable ya que los costos son más difíciles de vigilar y como se explicó antes, a través de ellos, existen muchas alternativas que sirven para rebajar la renta imponible. De todas formas, en el escenario base, la tasa óptima equivale a un royalty $\left(\mathrm{R}^{*}\right)$ de $15 \%$ sobre las ventas, monto mayor al 10\% que ha pagado CODELCO durante décadas sin dejar de ser rentable.

La columna PU indica la pérdida país por unidad (tonelada) producida en el proyecto. PU tiene la ventaja que al ser multiplicada por diferentes niveles de producción se puede actualizar a futuro. La pérdida anual PA expresa el mismo concepto amplificado al conjunto de la producción privada anual del país. En el escenario base, esta pérdida 'país es de US\$1.646 millones por año, la cual, sumando los 21 años de proyecto, crece a US\$ 34.566 millones.

Otra opción interesante es el precio de US\$ 3,0 porque coincide con el valor promedio 2006-2009, y con el período utilizado para promediar los costos de las empresas. Nótese que a los niveles de precio de US \$ 3,0, la tasa de retorno (TIR) del inversionista privado es 45\%. Esto significa que dos años bastan para casi duplicar el valor de la inversión. El impuesto óptimo IEM* que corresponde a ese precio es de 45 \% pero la tasa que se estableció en la Ley $\mathrm{N}^{\circ} 20.469$, es de solo un 9\% (Cuadro 1).

En realidad, si se cumpliera el pronóstico de algunos expertos citados por Cochilco, el precio del cobre esperado para el año 2012 alcanzaría US\$ 3,5. En tal caso, el modelo arroja una pérdida fiscal anual de US\$ 7.200 millones. Esta cantidad se acerca al costo fiscal del reciente terremoto del 24-F cifrado en US\$ 8.400 millones por ser uno de los más grandes del mundo. El efecto de las variaciones del precio sería más significativo si este modelo capturara que las condiciones externas que inciden en el precio del producto principal también influyen en el valor del subproducto.

Por su parte, el gráfico 2 permite comparar la tasa óptima (IEM*) con la tasa legal máxima después de impuesto de acuerdo a la Ley 20.469 (IEM). La brecha entre ambas, ilustrada con la flecha vertical, va creciendo a medida que aumenta el precio. Ello implica que la tasa legal no parece ser consistente con el criterio de mantener la tasa de rentabilidad privada. Desde luego que esta brecha seria aún mayor si se hubiese tomado como referencia legal el 4-5\% independiente del precio garantizado por invariabilidad tributaria. Por otra parte, este gráfico propone una curva que describe una ecuación de royalty óptima que resulta ser de tipo logarítmico con un significativo grado de ajuste (R2=98\%). 
Polis, Revista Latinoamericana, Volumen 13, No 37, 2014

\section{Cuadro 1: Pérdida país y tasas tributarias óptimas según precio del cobre.}

\begin{tabular}{|r|c|r|r|rrr|r|}
\hline \multicolumn{1}{|c|}{ P } & TIR & MOM & IEMx & \multicolumn{1}{c|}{ IEM* } & \multicolumn{1}{c|}{ R* } & \multicolumn{1}{c|}{ PU } \\
\hline US\$Lb & $\%$ & $\%$ Ventas & $\%$ RIOM & $\%$ RIOM & $\%$ Ventas & M US\$/ton & MM US\$/año \\
\hline $\mathbf{1 , 5}$ & $11 \%$ & $33 \%$ & $4,8 \%$ & $1 \%$ & $0 \%$ & 0 & 0 \\
\hline $\mathbf{1 , 6}$ & $14 \%$ & $38 \%$ & $4,9 \%$ & $11 \%$ & $4 \%$ & 1,60 & 245 \\
\hline $\mathbf{1 , 8}$ & $20 \%$ & $44 \%$ & $5,3 \%$ & $23 \%$ & $10 \%$ & 6,18 & 945 \\
\hline $\mathbf{2 , 0}$ & $25 \%$ & $50 \%$ & $5,9 \%$ & $30 \%$ & $15 \%$ & 10,76 & 1.645 \\
\hline $\mathbf{2 , 2}$ & $29 \%$ & $55 \%$ & $6,5 \%$ & $35 \%$ & $19 \%$ & 15,33 & 2.344 \\
\hline $\mathbf{2 , 4}$ & $34 \%$ & $58 \%$ & $6,8 \%$ & $38 \%$ & $22 \%$ & 19,91 & 3.044 \\
\hline $\mathbf{2 , 6}$ & $38 \%$ & $62 \%$ & $7,3 \%$ & $41 \%$ & $25 \%$ & 24,49 & 3.744 \\
\hline $\mathbf{2 , 8}$ & $41 \%$ & $64 \%$ & $7,6 \%$ & $43 \%$ & $28 \%$ & 29,06 & 4.444 \\
\hline $\mathbf{3 , 0}$ & $45 \%$ & $67 \%$ & $8,0 \%$ & $45 \%$ & $30 \%$ & 33,64 & 5.144 \\
\hline $\mathbf{3 , 2}$ & $49 \%$ & $69 \%$ & $8,2 \%$ & $46 \%$ & $32 \%$ & 38,22 & 5.843 \\
\hline $\mathbf{3 , 4}$ & $52 \%$ & $71 \%$ & $8,5 \%$ & $47 \%$ & $33 \%$ & 42,79 & 6.543 \\
\hline $\mathbf{3 , 6}$ & $55 \%$ & $72 \%$ & $8,7 \%$ & $48 \%$ & $35 \%$ & 47,37 & 7.243 \\
\hline $\mathbf{3 , 8}$ & $59 \%$ & $74 \%$ & $9,0 \%$ & $49 \%$ & $36 \%$ & 51,95 & 7.943 \\
\hline $\mathbf{4 , 0}$ & $62 \%$ & $75 \%$ & $9,1 \%$ & $49 \%$ & $37 \%$ & 56,52 & 8.642 \\
\hline
\end{tabular}

Fuente: Elaboración propia.

\section{Gráfico 2: Tasas Tributarias en función del Precio Promedio del Cobre.}

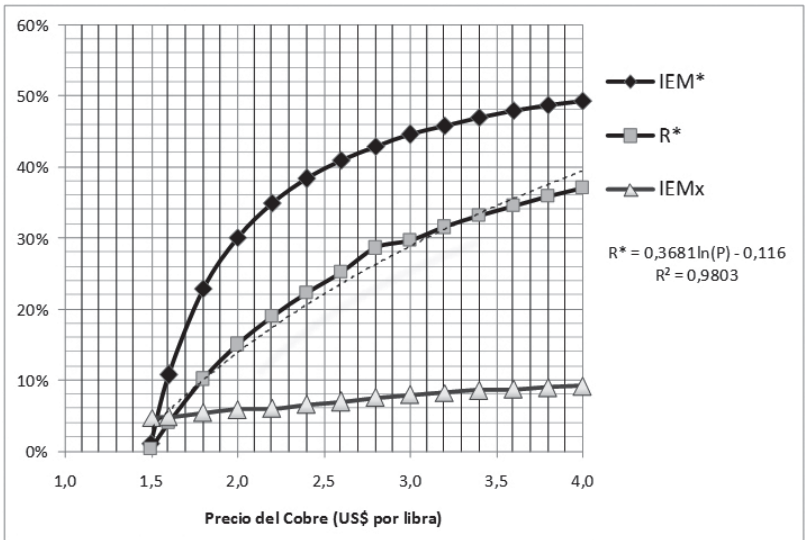

Fuente: Elaboración propia.

Posteriormente se sensibilizaron los costos de operación (Cuadro 2). El nivel mínimo de cero costos variables significa que el costo de operación del producto principal es cubierto por el ingreso neto de los sub-productos, situación que ocurre en CODELCO en los años investi- 
gados por Riesco y García Huidobro (2010). Por otra parte, la diferencia de pérdida anual país (PA) entre las filas del Cuadro 2 sugiere que al país cuesta cerca de US\$ 700 millones anuales cada US\$ 0.2 de mayor costo informado por las empresas. Luego, gastar en fiscalización, es fundamental. Alternativamente, estos datos sugieren que sería preferible negociar un royalty sub-óptimo antes que tener depender de la información de costos de la empresa.

\section{Cuadro 2: Pérdida país y tasas tributarias óptimas según costo operacional.}

\begin{tabular}{|c|c|c|c|c|c|c|c|c|}
\hline $\mathrm{Cv}_{\mathbf{v}}$ & Ct & TIR & MOM & IEMx & IEM $^{*}$ & $\mathrm{R}^{*}$ & PU & $\mathrm{PA}$ \\
\hline US S lb. & US S lb. & $\%$ & \%Ventas & \%RIOM & \% RIOM & $\%$ Ventas & M USS & MM USS \\
\hline 0,0 & 0,3 & $38 \%$ & $80 \%$ & $9,9 \%$ & $41 \%$ & $33 \%$ & 24,52 & 3.750 \\
\hline 0,2 & 0,5 & $34 \%$ & $70 \%$ & $8,2 \%$ & $38 \%$ & $27 \%$ & 19,70 & 3.012 \\
\hline 0,4 & 0,7 & $29 \%$ & $60 \%$ & $7,3 \%$ & $35 \%$ & $21 \%$ & 15,38 & 2.351 \\
\hline 0,6 & 0,9 & $25 \%$ & $50 \%$ & $5,4 \%$ & $30 \%$ & $15 \%$ & 10,72 & 1.639 \\
\hline 0,8 & 1,1 & $20 \%$ & $40 \%$ & $4,9 \%$ & $23 \%$ & $9 \%$ & 6,23 & 953 \\
\hline 1,0 & 1,3 & $14 \%$ & $30 \%$ & $4,8 \%$ & $11 \%$ & $3 \%$ & 1,66 & 253 \\
\hline 1,2 & 1,5 & $8 \%$ & $20 \%$ & $4,8 \%$ & $0 \%$ & $0 \%$ & - & - \\
\hline
\end{tabular}

Fuente: Elaboración propia.

En el Cuadro 3, se ha sensibilizado el modelo con diferentes niveles de Inversión Inicial. En la primera columna, la variable II (\%) indica que porcentaje de la Inversión Inicial del escenario base se simula. Así, el nivel 100\% representa al escenario base, es decir, inversión de US\$ 5,4 mil por tonelada de producción final de cobre. La varianza no ha sido demasiada; en la lista de 12 casos reales examinados, el proyecto más costoso se ubicó sólo un 30\% por sobre el escenario base, mientras que el proyecto más barato representaba un 37\% del mismo, de modo que ninguno de ellos alcanza diferencias tan extremas como las simuladas. En este Cuadro 3 se evidencia que incluso con una Inversión Inicial el doble de onerosa del escenario base (200\%), el proyecto soporta una rentabilidad TIR de 14\%. En tal situación, el IEM óptimo debería ser de $13 \%$; cifra bastante superior a los actuales niveles de tasa media IEM efectiva antes impuesto de 5,1\% para un MOM de 45\%-50\%.

Finalmente, se sensibilizan cambios en la tasa de rentabilidad exigida por los propietarios. Como se observa en el Cuadro 4, incluso, si la rentabilidad exigida fuese de $22 \%$, la tasa de impuesto óptima (IEM*) es de $10 \%$, porcentaje que duplica la tasa antes impuesto de la actual Ley 20.469 de 5,4\%. Otro resultado interesante es que, un aumento de $1 \%$ en la tasa de descuento a partir del escenario base, ocasiona una disminución de $2 \%$ en la tasa IEM y de $1 \%$ en el royalty óptimo. 
Polis, Revista Latinoamericana, Volumen 13, No 37, 2014

\section{Cuadro 3: Pérdida país y tasas tributarias óptimas según inversión inicial.}

\begin{tabular}{|c|c|c|c|c|c|c|c|}
\hline II & TIR & MOM & IEMx & IEM & R* & PU & PA \\
\hline$\%$ base & $\%$ & $\%$ Ventas & $\%$ RIOM & $\%$ RIOM & $\%$ Ventas & M USS & MM US\$ \\
\hline $\mathbf{2 0} \%$ & $92 \%$ & $54 \%$ & $5,4 \%$ & $52 \%$ & $28 \%$ & 21,54 & 3.293 \\
\hline $\mathbf{4 0} \%$ & $54 \%$ & $53 \%$ & $5,4 \%$ & $46 \%$ & $24 \%$ & 18,47 & 2.824 \\
\hline $\mathbf{6 0} \%$ & $39 \%$ & $52 \%$ & $5,4 \%$ & $40 \%$ & $21 \%$ & 15,51 & 2.371 \\
\hline $\mathbf{8 0} \%$ & $30 \%$ & $51 \%$ & $5,4 \%$ & $35 \%$ & $18 \%$ & 13,07 & 1.999 \\
\hline $100 \%$ & $25 \%$ & $50 \%$ & $5,4 \%$ & $30 \%$ & $15 \%$ & 10,72 & 1.639 \\
\hline $\mathbf{1 2 0} \%$ & $21 \%$ & $49 \%$ & $5,4 \%$ & $26 \%$ & $13 \%$ & 8,86 & 1.355 \\
\hline $\mathbf{1 4 0} \%$ & $18 \%$ & $48 \%$ & $5,4 \%$ & $21 \%$ & $10 \%$ & 6,67 & 1.020 \\
\hline $\mathbf{1 6 0} \%$ & $16 \%$ & $47 \%$ & $5,4 \%$ & $17 \%$ & $8 \%$ & 4,96 & 758 \\
\hline $\mathbf{1 8 0} \%$ & $16 \%$ & $46 \%$ & $5,4 \%$ & $18 \%$ & $8 \%$ & 5,24 & 801 \\
\hline $\mathbf{2 0 0} \%$ & $14 \%$ & $45 \%$ & $5,4 \%$ & $13 \%$ & $6 \%$ & 3,24 & 495 \\
\hline
\end{tabular}

Fuente: Elaboración propia.

\section{Cuadro 4: Pérdida país y tasas tributarias óptimas según tasa de retorno exigida.}

\begin{tabular}{|r|r|r|r|r|r|r|c|}
\hline Tasa & \multicolumn{1}{c|}{ TIR } & \multicolumn{1}{c|}{ MOM } & \multicolumn{1}{c|}{ IEMx } & \multicolumn{1}{c|}{ IEM } & \multicolumn{1}{c|}{ R } & \multicolumn{1}{c|}{ PU } & \multicolumn{1}{c|}{ PA } \\
\hline$\%$ & \multicolumn{1}{c|}{$\%$} & $\%$ Ventas & \multicolumn{1}{c|}{ RIOM } & $\%$ RIOM & $\%$ Ventas & M US\$ & MM USS \\
\hline 6,0 & $25 \%$ & $50 \%$ & $5.4 \%$ & $39 \%$ & $20 \%$ & 14,5 & 2.216 \\
\hline 8,0 & $25 \%$ & $50 \%$ & $5.4 \%$ & $36 \%$ & $18 \%$ & 13,24 & 2.024 \\
\hline 10,0 & $25 \%$ & $50 \%$ & $5.4 \%$ & $33 \%$ & $17 \%$ & 11,98 & 1.832 \\
\hline 12,0 & $25 \%$ & $50 \%$ & $5.4 \%$ & $30 \%$ & $15 \%$ & 10,72 & 1.639 \\
\hline 14,0 & $25 \%$ & $50 \%$ & $5.4 \%$ & $26 \%$ & $13 \%$ & 9,04 & 1.383 \\
\hline 16,0 & $25 \%$ & $50 \%$ & $5.4 \%$ & $22 \%$ & $11 \%$ & 7,37 & 1.127 \\
\hline 18,0 & $25 \%$ & $50 \%$ & $5.4 \%$ & $18 \%$ & $9 \%$ & 5,69 & 870 \\
\hline 20,0 & $25 \%$ & $50 \%$ & $5.4 \%$ & $14 \%$ & $7 \%$ & 4,01 & 614 \\
\hline 22,0 & $25 \%$ & $50 \%$ & $5.4 \%$ & $10 \%$ & $5 \%$ & 2,34 & 358 \\
\hline
\end{tabular}

Fuente: Elaboración propia.

Algunas observaciones finales. A menudo las empresas emplean para evaluar sus proyectos de inversión la llamada tasa de costo capital promedio ponderado que combina la rentabilidad exigida por el propietario con la de los tenedores de deuda. Se necesita mayor investigación acerca de cómo los resultados habrían cambiado con ese método. 


\section{Conclusiones}

En este artículo se muestra que el cobre genera importantes rentas en Chile. Ello justifica la necesidad de mejorar la estructura tributaria para que el país pueda capturar la renta que hoy reciben las empresas multinacionales. El impuesto óptimo que aquí se propone, depende del precio del cobre. Se presenta bajo la forma de un impuesto específico a la minería (IEM) sobre la utilidad operacional o como royalty sobre las ventas. Ambos tienen sus ventajas ya que el primero permite adecuarse a las condiciones de rentabilidad de las diversas empresas, mientras que el segundo, permite evitar la elusión del impuesto a través de costos artificiales.

Para este efecto, se simuló con un proyecto de cobre representativo, al cual se impuso una rentabilidad privada (TIR) sobre el capital aportado por los dueños, considerada normal, de $12 \%$ por año. Actualmente, para un precio de US\$ 3,0 y costos de US\$ 1,5 esta TIR, asciende a cerca de $38 \%$ al año; esto significa que al cabo de tres años se tiene recuperada la inversión inicial y además se ha ganado otro proyecto de similar tamaño al original. Esta elevada tasa de rentabilidad probablemente es incluso mayor, considerando los diferentes medios que citamos, para rebajar impuestos.

En nuestro escenario base, con un precio de US\$2, nuestro modelo recomienda un IEM de 30\% sobre la renta operacional, equivalente a un royalty sobre las ventas de $15 \%$. Esta cifra es bastante superior a las tasas vigentes de IEM, de $6 \%$ para ese precio o de $4-5 \%$ para las empresas que utilizan la estabilidad tributaria de arrastre. Estas últimas tasas provocan una pérdida anual fiscal del orden de US\$ 1.645 millones para el Estado, sin considerar las externalidades negativas y positivas de la gran minería.

En los dos últimos años, las empresas señalan que sus costos operacionales estarían un 50\% por sobre la estimación empleada en este trabajo de US\$ 0.9 aproximadamente. Sin embargo, también en los últimos años (2006-2009) el precio promedio del cobre ha estado más cerca de los US\$ 3 que de US\$2. En tal caso, el IEM óptimo recomendado de 30\%, parece plenamente razonable. En cualquier caso, en este trabajo, se ofrecen diferentes tasas de impuesto, en función de las variaciones de precios de venta, de costos de producción de las empresas, de costos de inversión y tasas de descuento.

Por otro lado, el modelo sugiere que bastan diez centavos de dólar de mayor costo de operación (o menor precio del cobre) para que el Estado pierda US\$ 350 millones de dólares anuales por no aplicar el impuesto óptimo. El resultado ratifica dos cosas: la importancia de fiscalizar las declaraciones tributarias de las empresas y la conveniencia de tener un impuesto óptimo.

Otro resultado que debería ser preocupante para la política pública, es la diferencia creciente entre la tasa recomendada y la tasa efectiva de arrastre, vis a vis el incremento del precio del cobre. Para evitar aquello, el royalty debería seguir una curva logística con el precio. La escasez progresiva de este recurso no renovable podría incrementar su valor y el costo 
de la pérdida país con la actual legislación.

Finalmente, hay algunas debilidades de esta investigación que podrían estimular nuevas indagaciones. Falta construir algún modelo aparte, para justificar la tasa de rentabilidad normal para el sector privado. Por otra parte, se requiere conocer el convenio de estabilidad tributaria que firmaron las grandes empresas mineras caso por caso para afinar el cálculo de la pérdida país. Esta pérdida también podría ser afinada calibrando el modelo con información de recaudación proveniente directamente del Estado. Asimismo, las regresiones econométricas para estimar costos podrían ser mejoradas con nueva información actualizada. No obstante lo anterior, la principal dificultad para estimar costos es la escasa confiabilidad de la información financiera de las empresas para efectos tributarios.

\section{Cuadro 1 Anexo: Información principales operaciones mineras, año 2011.}

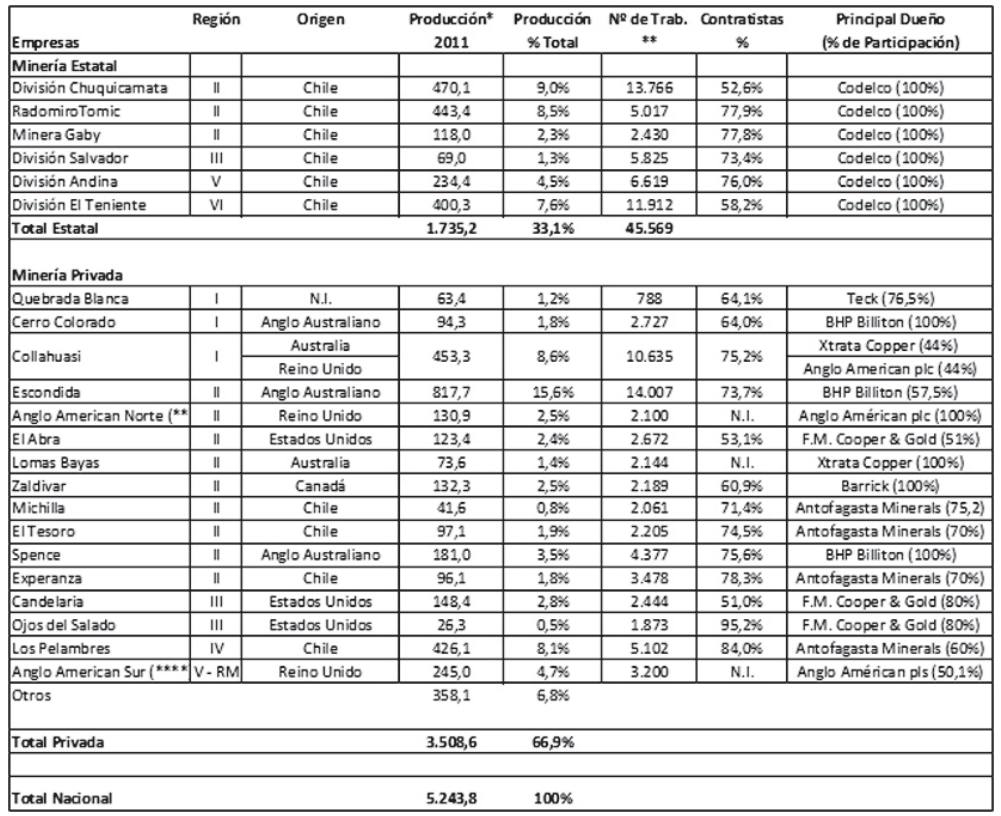

Fuente: Elaboración propia a partir de COCHILCO y Consejo Minero.

(*) La producción está medida en miles de toneladas de cobre fino.

(**) Es la suma de trabajadores contratados por la empresa, más los trabajadores contratistas.

Trabajadores al 31 de Diciembre de 2011.

$(* * *)$ El Soldado y Los Bronces.

$(* * * *)$ Mantos Blancos, Manto Verde. 


\section{Cuadro 2 Anexo: Impuesto Específico a la Minería según Ley 20.469.}

\begin{tabular}{|c|c|c|c|c|c|c|c|}
\hline A & B & $\mathrm{C}$ & D & $\mathrm{E}=\mathrm{B} * \mathrm{D}$ & $\mathrm{F}=\mathrm{A}+\mathrm{E}$ & $\begin{array}{c}\mathrm{G}=\mathrm{E} / \mathrm{F} \\
\text { (Método 1) }\end{array}$ & $\begin{array}{l}\mathrm{H}=\mathrm{D} /(1-\mathrm{D}) \\
\text { (Método 2) }\end{array}$ \\
\hline $\begin{array}{l}\text { MOM } \\
\text { Después } \\
\text { IEM }\end{array}$ & $\begin{array}{l}\text { Mediana } \\
\text { Intervalo }\end{array}$ & $\begin{array}{c}\text { Tasa } \\
\text { Marginal } \\
\text { después } \\
\text { IEM }\end{array}$ & $\begin{array}{c}\text { Tasa efectiva } \\
\text { después } \\
\text { IEM }\end{array}$ & IEM a pagar & $\begin{array}{l}\text { MOM } \\
\text { antes } \\
\text { IEM }\end{array}$ & $\begin{array}{c}\text { Tasa } \\
\text { efectiva } \\
\text { antes } \\
\text { IEM }\end{array}$ & $\begin{array}{l}\text { Tasa } \\
\text { efectiva } \\
\text { antes } \\
\text { IEM }\end{array}$ \\
\hline$>x \leq$ & $\%$ Ventas & $\%$ & \%RIOM & \%RIOM & $\%$ Ventas & \%RIOM & \%RIOM \\
\hline $0-35$ & $32,5 \%$ & $5,0 \%$ & $5,0 \%$ & 1,63 & $34,1 \%$ & $4,8 \%$ & $4,8 \%$ \\
\hline $35-40$ & $37,5 \%$ & $8,0 \%$ & $5,2 \%$ & 1,95 & $39,5 \%$ & $4,9 \%$ & $4,9 \%$ \\
\hline $40-45$ & $42,5 \%$ & $10,5 \%$ & $5,7 \%$ & 2,30 & $44,8 \%$ & $5,4 \%$ & $5,4 \%$ \\
\hline $45-50$ & $47,5 \%$ & $13,0 \%$ & $6,3 \%$ & 2,71 & $50,2 \%$ & $5,9 \%$ & $5,9 \%$ \\
\hline $50-55$ & $52,5 \%$ & $15,5 \%$ & $7,1 \%$ & 3,73 & $56,2 \%$ & $6,6 \%$ & $6,6 \%$ \\
\hline $55-60$ & $57,5 \%$ & $18,0 \%$ & $7,9 \%$ & 4,54 & $62,0 \%$ & $7,3 \%$ & $7,3 \%$ \\
\hline $60-65$ & $62,5 \%$ & $21,0 \%$ & $8,9 \%$ & 5,56 & $68,1 \%$ & $8,2 \%$ & $8,2 \%$ \\
\hline 65.70 & $67,5 \%$ & $24,0 \%$ & $9,9 \%$ & 6,68 & $74,2 \%$ & $9,0 \%$ & $9,0 \%$ \\
\hline $70-75$ & $72,5 \%$ & $27,5 \%$ & $11,0 \%$ & 7,98 & $80,5 \%$ & $9,9 \%$ & $9,9 \%$ \\
\hline $75-80$ & $77,5 \%$ & $31,0 \%$ & $12,2 \%$ & 9,46 & $87,0 \%$ & $10,9 \%$ & $10,9 \%$ \\
\hline $80-85$ & $82,5 \%$ & $34,5 \%$ & $13,4 \%$ & 11,06 & $93,6 \%$ & $11,8 \%$ & $11,8 \%$ \\
\hline$>85$ & $87,50 \%$ & $14,0 \%$ & $14,0 \%$ & 12,25 & $99,8 \%$ & $12,3 \%$ & $12,3 \%$ \\
\hline
\end{tabular}

Fuente: Elaboración Propia a partir de columna E. 


\section{Bibliografía}

Alcayaga, J. (2005), Manual del Defensor del Cobre. Ediciones Tierra Mía Limitada. http://druzhba.se/druzhba/articulos/cobre_chileno_ja.pdf. Consultado el 26.03.2011

Cademartori, J. (2005), Análisis Tributación Sector Minero, Segunda Región: Periodo 1999-2000. Departamento Economía UCN,Antofagasta de Chile.

CEPAL (2012), Cambio Estructural para la Igualdad. Una Visión Integrada para el Desarrollo. Trigésimo Cuarto Periodo de Sesiones de la CEPAL. San Salvador. 27 al 31 de Agosto.

COCHILCO (2011), Precio real y nominal del cobre refinado. 1950-2008. Recuperado el 11 de Abril de 2011, de: http://www.cochilco.cl/.../ Precio_nominal_y_real_del_cobre_refinado_1950-2008.xls.

Ferreira, F.; Messina, J.; Rigolini J.; López-Calva L.; Lugo M. y Vakis R. (2013), Economic Mobility and the Rise of the Latin American Middle Class. The World Bank, Washington D.C.

González, J. (2004), “Las empresas mineras y la buena fe contractual”. Estudios Públicos, 95 (invierno 2004), Santiago.

Leiva, S. (2009), “La subcontratación en la minería enChile: elementos teóricospara el análisis”. Polis $N^{\circ} 19$, Revista Latinoamericana, Santiago.

Leturia, F. y Merino A. (2004), “Tributación y Minería en Chile: Antecedentes para un debate informado”. Estudios Públicos, $N^{\circ} 95$ (invierno 2004).Santiago.

Meller, P. (2002), “El Cobre Chileno y la Política Minera”. En Meller, P. (2002) Dilemas y Debates en torno al Cobre. Santiago de Chile.

Otto, J.; Batarseh, M.L.y Cordes, J. (2000), GlobalMining Taxation Comparative Study. Institute for Global Resources Policy and Management, Colorado School of Mines, USA.

Riesco M. y GarcíaHuidobro, G. (2010), Tributación y Ganancias de la Gran Minería en Chile: 2005-2009. Centro de Estudios de Desarrollo Alternativo, Santiago.

Recibido: 14.12.2012

Aceptado: 24.07.2013 